\title{
SUBGAP ABSORPTION IN CONJUGATED POLYMERS
}

M. Sinclair*, C. H. Seager*, D. McBranch**, A.J. Heeger** af AOG.L. Baker*** *Sandia National Laboratories, Albuquerque, NM ** University of California, Santa Barbara, CA SAND- $-90-3023 \mathrm{C}$ ***Bell Communications Research, Red Bank, NJ

DE91 012655

\section{ABSTRACT}

Along with $\chi^{(3)}$, the magnitude of the optical absorption in the transparent window below the principal absorption edge is an important parameter which will ultimately determine the utility of conjugated polymers in active integrated optical devices. With an absorptance sensitivity of $<10^{-5}$, Photothermal Deflection Spectroscopy (PDS) is ideal for determining the absorption coefficients of thin films of "transparent" materials. We have used PDS to measure the optical absorption spectra of the conjugated polymers poly[1,4-phenylenevinylene] (and derivitives) and polydiacetylene-4BCMU in the spectral region from $0.55 \mathrm{eV}$ to $3 \mathrm{eV}$. Our spectra show that the shape of the absorption edge varies considerably from polymer to polymer, with polydiacetylene-4BCMU having the steepest absorption edge. The minimum absorption coefficients measured varied somewhat with sample age and quality, but were typically in the range $1 \mathrm{~cm}^{-1}$ to $10 \mathrm{~cm}^{-1}$. In the region below $1 \mathrm{eV}$, overtones of $\mathrm{C}-\mathrm{H}$ stretching modes were observed, indicating that further improvements in transparency in this spectral region might be achieved via deuteration or fluorination.

\section{INTRODUCTION}

One of the principal challenges associated with the advancement of integrated optics is the development of nonlinear optical materials for use in active elements. Conjugated polymers, because of their large nonlinear optical susceptibilities, have been recognized as a class of materials possessing great potential for use in these applications [1]. However, in contrast to the large amount of research that has been done on their nonlinear optical properties, the important subject of optical losses has received relatively little attention $[2,3]$. In order to fully evaluate conjugated polymers for guided wave applications, it is essential that the mechanisms of optical absorption be understood.

Attenuation of a guided optical wave can arise from a number of intrinsic and extrinsic sources, and can have detrimental effects on the performance of a device element. Onephoton absorption arising from overtones of lattice modes and the low energy tail of the principal electronic absorption edge; as well as two-photon absorption [4], are examples of intrinsic loss mechanisms. Extrinsic loss mechanisms include scattering from material inhomogeneities and dimensional nonuniformities of the waveguide; and absorption losses due to impurities. While extrinsic loss mechanisms can, in principle, be eliminated through improved material processing, intrinsic losses can only be reduced by altering the nature of the material (at the risk of decreasing the magnitude of the optical nonlinearities).

When significant losses are present, the distance over which a useful amount of light may be propagated is limited. Thus, the number of active optical elements that a light wave can encounter before it must be detected or amplified decreases with increasing attenuation. A less obvious problem is that absorption of optical energy in a dispersive (i.e. phase shifting) 


\section{DISCLAIMER}

This report was prepared as an account of work sponsored by an agency of the United States Government. Neither the United States Government nor any agency Thereof, nor any of their employees, makes any warranty, express or implied, or assumes any legal liability or responsibility for the accuracy, completeness, or usefulness of any information, apparatus, product, or process disclosed, or represents that its use would not infringe privately owned rights. Reference herein to any specific commercial product, process, or service by trade name, trademark, manufacturer, or otherwise does not necessarily constitute or imply its endorsement, recommendation, or favoring by the United States Government or any agency thereof. The views and opinions of authors expressed herein do not necessarily state or reflect those of the United States Government or any agency thereof. 


\section{DISCLAIMER}

Portions of this document may be illegible in electronic image products. Images are produced from the best available original document. 
device can result in a thermally induced refractive index change that could dominate the optically induced refractive index change. Optical absorption can also lead to damage or degradation of the nonlinear material. Finally, scattering of the guided optical wave could cause cross-talk between nearby guides.

Detailed investigations of the optical losses of the earliest conjugated polymers such as polyacetylene and polythiophene were largely impractical because films of these materials tended to be of very poor optical quality, making assessment of the relative influence of factors such as scattering and impurity absorption on experimental findings difficult. More recently, however, there has been an increased emphasis on processability of conjugated polymers, and as a result, there have been significant improvements in film quality. In particular, the development of materials which are soluble in organic solvents, coupled with the application of solution processing techniques (such as solution filtering, spin-casting, etc.), has led to the production of thin films of vastly improved quality [5]. With these better films, detailed studies of optical losses become possible. In this paper, we describe the results of Photothermal Deflection Spectroscopy measurements of the subgap absorption of solution processed conjugated polymers.

\section{EXPERIMENTAL}

In Photothermal Deflection Spectroscopy (PDS), the absorption of a material is determined by measuring the temperature rise of the sample when it is irradiated by an optical beam of known intensity [6]. To record the temperature rise, the sample is immersed in a liquid and the beam from a Helium-Neon laser is propagated parallel to the front surface of the material in the region illuminated by the heating beam. Any change in the temperature at the surface of the sample will result in the formation of a thermal gradient in the liquid immediately adjacent to the surface of the sample. Because the refractive index of the liquid is temperature dependent, the induced refractive index gradient will cause an angular deflection of the laser beam. The amount of heating light absorbed by the sample may be deduced by measuring the magnitude of this deflection, and comparing it to the deflection induced by a totally absorbing sample.

In practice, the samples were suspended in a $1 \mathrm{~cm}$ cuvette filled with Fluorinert (FC75). The beam from a stabilized He-Ne laser was focussed with a cylindrical lens so that the beam waist occured at the center of the cell. After traversing the sample cell, the angular deflection of the beam was measured using a knife-edge detector. In addition, a portion of the He-Ne beam was split off prior to the sample cell and directed onto an identical detector for use in reducing noise due to amplitude and beam pointing fluctuations of the laser. The heating beam was obtained using a tungsten-halogen lamp, mechanical chopper, and grating monochromator; and lock-in detection was used to measure the deflection of the $\mathrm{He}-\mathrm{Ne}$ beam. For further noise reduction, the entire apparatus was arranged on a vibration isolated table, and the sample chamber was enclosed in an air-tight box. The spectral range of our spectrometer extends from $0.55 \mathrm{eV}$ to $3 \mathrm{eV}$ with an absorptance sensitivity of $<10^{-5}$.

The choice of Fluorinert FC-75 was dictated by the need to use a liquid which is a nonsolvent for the (soluble) conjugated polymers, but which possesses a high degree of transparency in the spectral region of interest. The samples were prepared by spin-casting thin films onto fused silica substrates. Whenever possible, sample handling procedures were 
performed in inert environments. Prior to mounting the samples in the cuvette, helium was bubbled through the fluorinert to reduce the concentration of dissolved oxygen; and after mounting, the sample cell was hermetically sealed. Subsequent to measuring the absorptance spectra, the thickness of the samples were measured using a Sloan Dektak, and the absorption spectra were calculated.

\section{RESULTS AND DISCUSSION}

In general, the PDS spectra for a given polymer varied somewhat from sample to sample. This was particularly true in the initial stages of the experiment before it was realized that exposure to above band gap light (particularly in the presence of oxygen) can lead to significant alteration of the subgap absorption spectra. For subsequent samples, care was taken to minimize exposure to air and light. With reasonable care, the variations from sample to sample were typically no more than $\pm 25 \%$.

Fig. 1 displays the PDS spectra of a thin film of poly[1,4-phenylene-vinylene] (PPV). The film was prepared by depositing the precursor polymer from aqueous solution, followed by heating to convert it to PPV [7]. For PPV, the slope of the absorption edge is largest at the highest energies and has an abrupt change to a smaller slope at about $2.3 \mathrm{eV}$. Below this energy the absorption gradually decreases to a value of about $6 \mathrm{~cm}^{-1}$, which is the minimum absorption observed for this material. In the low energy portion of the spectrum, there is a pronounced peak at $0.74 \mathrm{eV}$ which we believe is the first overtone of the $\mathrm{C}-\mathrm{H}$ stretching mode $\left(\nu_{2}^{\mathrm{C}-\mathrm{H}}\right)$. The smaller peak at $0.88 \mathrm{eV}$ is due to the stretching plus bending combination overtone $\left(\nu_{2}^{\mathrm{C}-\mathrm{H}}+\delta^{\mathrm{C}-\mathrm{H}}\right)$ with some contribution from the first overtone of the substrate $\mathrm{O}-\mathrm{H}$ stretching mode $\left(\nu_{2}^{\mathrm{O}-\mathrm{H}}\right)$, since both of these absorptions are expected to appear at about the same energy.

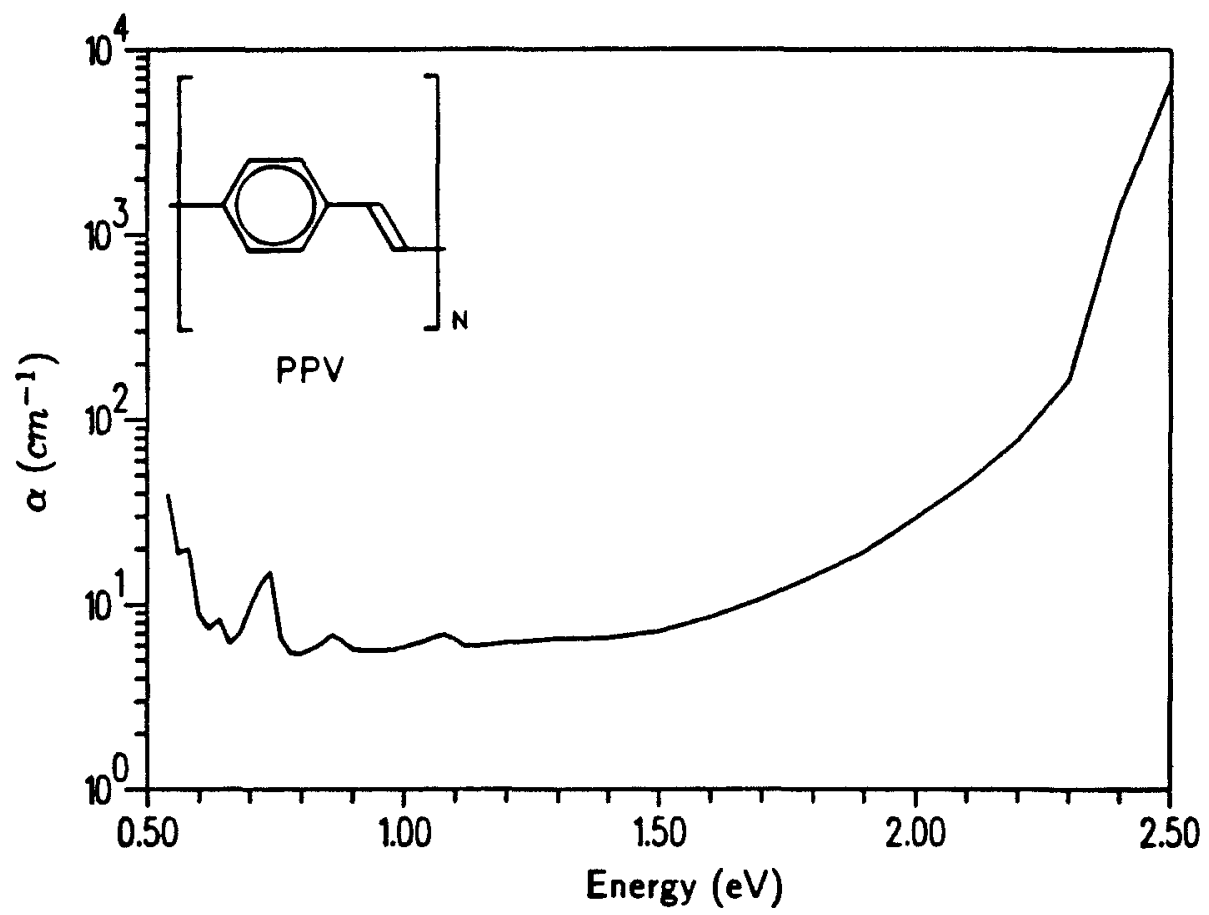

Fig. 1. Structure and PDS spectrum of PPV. 


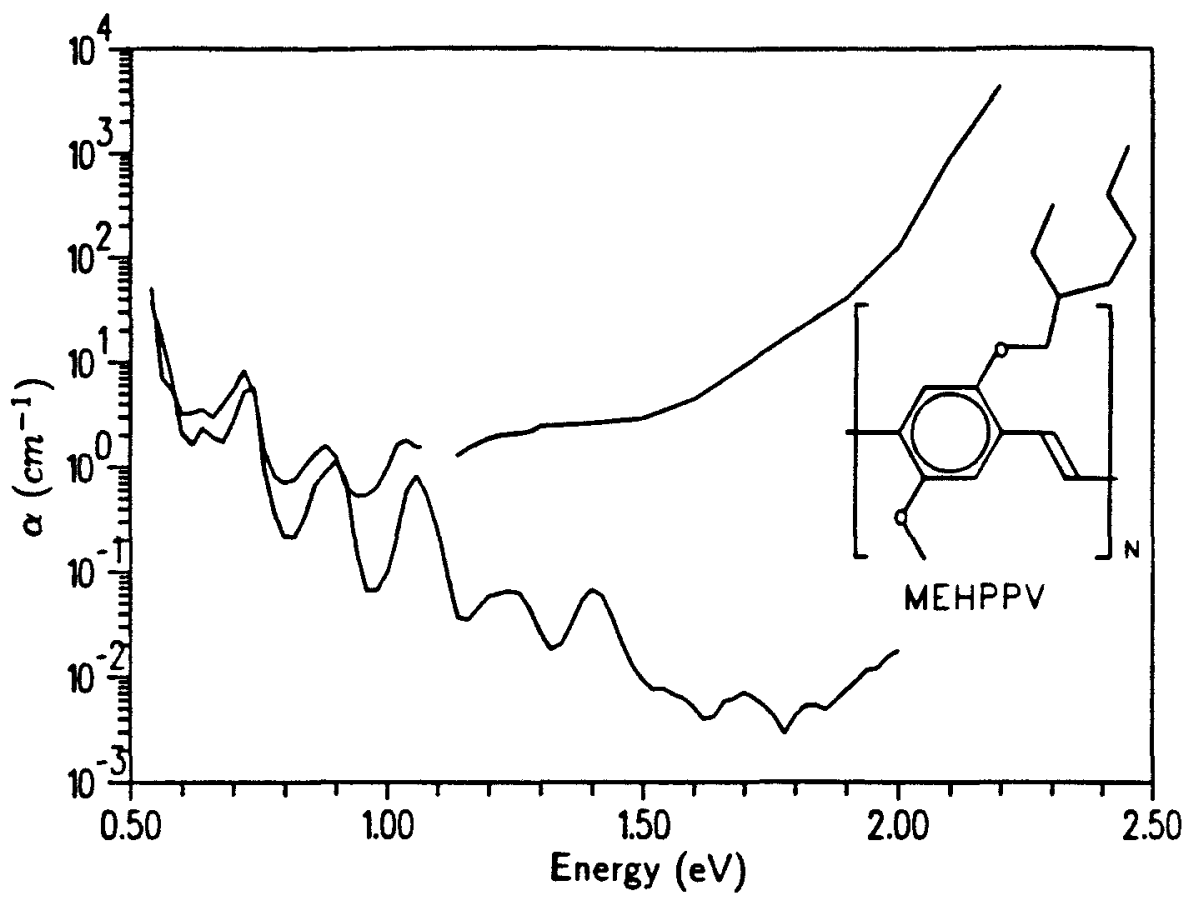

Fig. 2. Upper curve: PDS spectrum of MEHPPV. Lower curve: PDS spectrum of PMMA.

The upper curve in Fig. 2 is the PDS spectrum of a thin film of poly[2-methoxy, 5(2'-ethyl-hexoxy)-PPV] (MEHPPV) prepared by spin-casting from $\mathrm{m}$-xylenes. The band edge of MEHPPV is red shifted by about $0.3 \mathrm{eV}$ relative to that of PPV due to the addition of the alkoxy side chains [8]. The minimum absorption coefficient observed for MEHPPV $\left(0.7 \mathrm{~cm}^{-1}\right)$ occurs at $0.95 \mathrm{eV}$, and, once again, overtone features appear in the low energy portion of the spectrum. An interesting feature of the PDS spectrum of MEHPPV is the broad featureless absorption which is visible as a shoulder at $1.3 \mathrm{eV}$. We believe that this is due to residual (long-lived) photoexcitations generated by light exposure during sample handling as well as the high energy portion of the PDS run. This observation is supported by the photoinduced absorption data presented in a companion paper [9], as well as the data of Voss et al. [8]. If this is indeed the case, then for maximum subgap transparency, samples of this material should be protected from photons with energy above the $\pi-\pi^{*}$ transition.

To further investigate the role of $\mathrm{C}-\mathrm{H}$ overtones, we measured the PDS spectrum of the nonconjugated polymer poly[methylmethacrylate] (lower curve in Fig. 2). In the nearinfrared, the absorption spectrum of this material is known to be dominated by $\mathrm{C}-\mathrm{H}$ overtones [10]. We have converted our raw absorptance data to an absorption spectrum by scaling our data so that the magnitude of the overtone absorption at $0.74 \mathrm{eV}$ agreed with the data of Kaino et al. [10]. The close similarity between the spectra of MEHPPV and PMMA in the spectral region below $1.1 \mathrm{eV}$, further supports the assignments of the $\mathrm{C}-\mathrm{H}$ overtone absorptions described above. From the PDS spectrum of PMMA, we can see that the magnitude of the $\mathrm{C}-\mathrm{H}$ absorption falls quickly with increasing photon energy, and in the spectral region from $1.1 \mathrm{eV}$ to $2 \mathrm{eV}, \mathrm{C}-\mathrm{H}$ absorption contributes less than $0.1 \mathrm{~cm}^{-1}$. Thus, we conclude that, although $\mathrm{C}-\mathrm{H}$ overtones are observable at low energies, the residual subgap absorption above $\sim 1 \mathrm{eV}$ in MEHPPV (and PPV) is dominated by other mechanisms. 


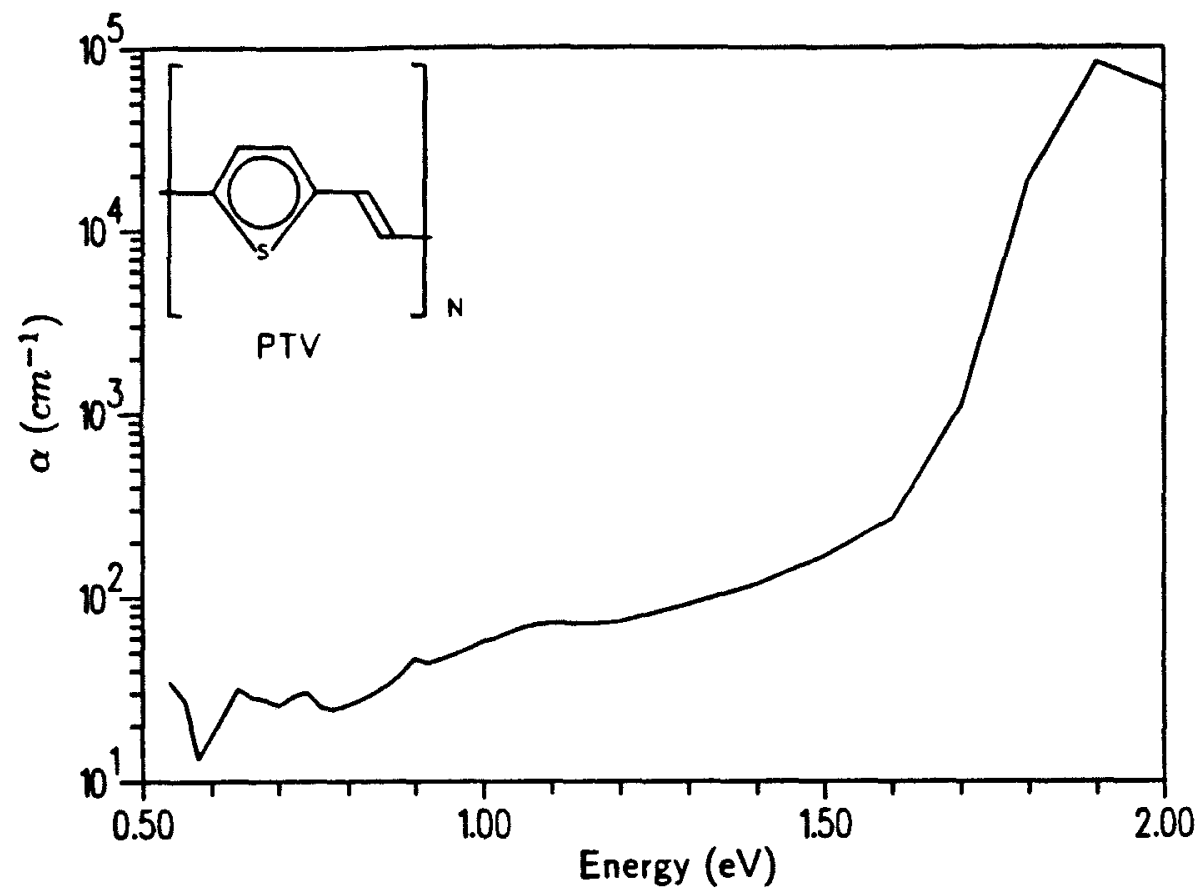

Fig. 3. Structure and PDS spectrum of PTV.

The PDS spectrum of poly[2,5-thienylene-vinylene] (PTV) is displayed in Fig. 3. As in the case of PPV, this film was prepared by solution deposition of a precursor polymer and subsequent conversion to the final form [11]. The absorption edge of PTV is significantly red shifted relative to that of $P P V$, and the minimum absorption observed is $20-30 \mathrm{~cm}^{-1}$. As in the case of MEHPPV, a broad subgap absorption peak is observed (this time occuring at about $1.1 \mathrm{eV}$ ) which may be due to residual photoexcitations generated in the sample handling and measurement process.

Finally, the subgap optical spectrum of a film of poly[5,7-dodecadiyne-1,12-diolbis ( $n$ butoxycarbonylmethylurethane)] (PDA-4BCMU), is presented in Fig. 4. This film was

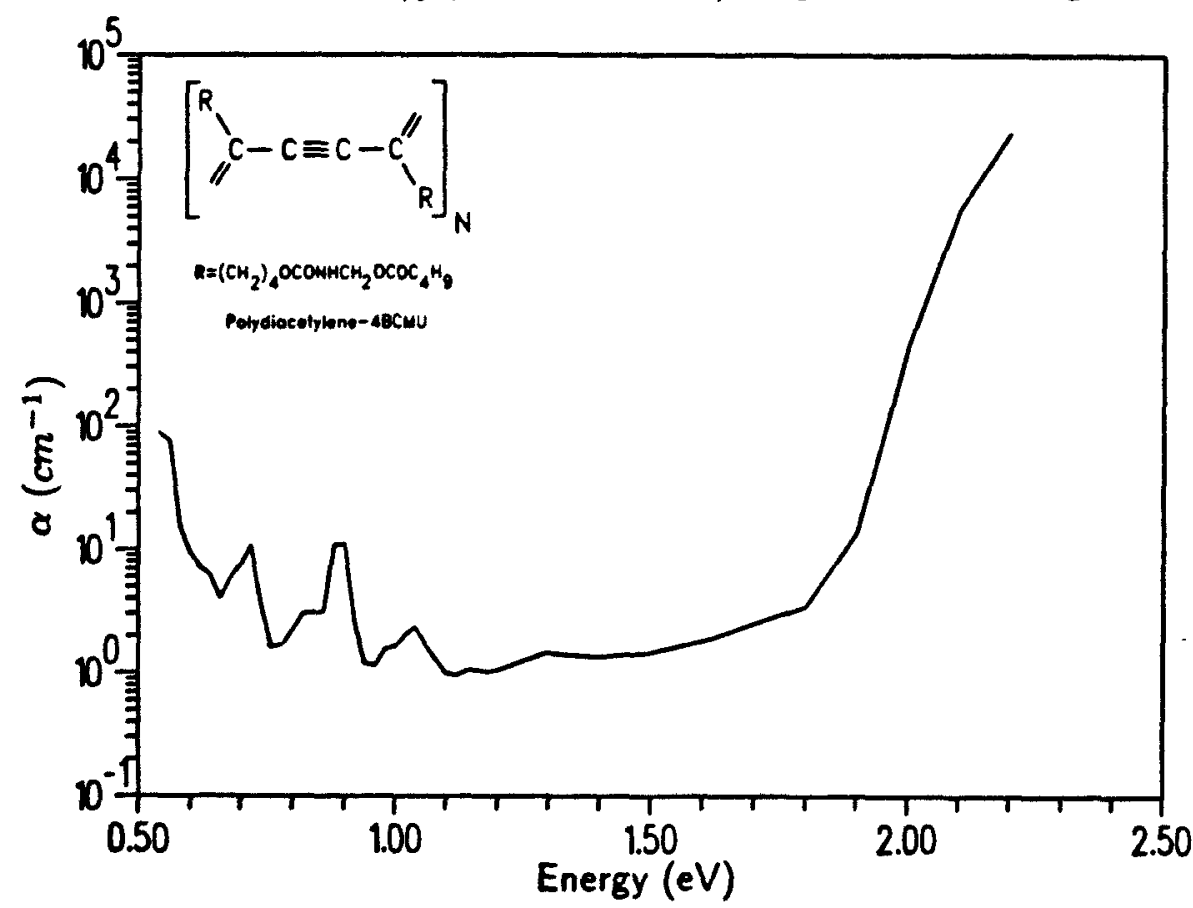

Fig. 4. Structure and PDS spectrum of polydiacetylene-4BCMU. 
prepared by spin-casting from cyclopentanone [5]. Of all the conjugated polymers we have measured, PDA-4BCMU possesses the sharpest absorption edge, with the absorption coefficient falling quickly to a value of about $1 \mathrm{~cm}^{-1}$. At lower energies, overtone features are again observed.

From these results, we conclude that the most serious source of subgap absorption in conjugated polymers is the low energy tail of the principle absorption edge, which decreases very slowly with decreasing photon energy. Although the origin of this tail is not presently known, the following mechanisms may contribute: inhomogeneous broadening due to a distribution of chain lengths and environments; large differences in ground and excited state geometries (i.e. vibronic effects); impurity absorption; interchain interactions; and the absorption tails of broad higher-lying absorptions. Current investigations are directed towards identifying the contribution of each of these mechanisms to the subgap absorption.

\section{CONCLUSION}

We have measured the subgap optical absorption of conjugated polymers using Photothermal Deflection Spectroscopy. At the lowest photon energies, absortions due to $\mathrm{C}-\mathrm{H}$ overtones are observed. By comparing with the $\mathrm{C}-\mathrm{H}$ overtone spectrum of PMMA, we conclude that for most integrated optical applications overtone absorption will not present a significant problem. In contrast, we find that the presence of a low energy tail of the principle absorption edge severely limits the transparency of the conjugated polymers we have measured. The minimum absorpion coefficients observed ranged from $1 \mathrm{~cm}^{-1}(\sim 4 \mathrm{~dB} / \mathrm{cm})$ to $10 \mathrm{~cm}^{-1}(40 \mathrm{~dB} / \mathrm{cm})$. The origin of this tail must be identified in order to continue to develop conjugated polymers for guided wave applications.

Acknowledgements: We thank W.R. Bayless for assistance in making the PDS measurements.

\section{REFERENCES}

1. Nonlinear Optical Properties of Polymers, edited by A.J. Heeger, J. Orenstein, and D. Ulrich (Mater. Res. Soc. Proc. 109, Pittsburgh, PA, 1988).

2. M. Thakur, R.C. Frye, and B.I. Greene, Appl. Phys. Lett. 56, 1187 (1990).

3. W. Krug, E. Miao, M. Derstine, and J. Valera, JOSA B6, 726 (1989).

4. P.D. Townsend, W.-S. Fann, S. Etemad, G.L. Baker, Z. G. Zoos, and P.C.M. McWilliams, (Chem. Phys. Lett., submitted).

5. P.D. Townsend, G.L. Baker, N.E. Sclotter, C.F. Klausner, and S. Etemad, Appl. Phys. Lett. 53, 1782, (1988).

6. W.B. Jackson, N. Amer, A.C. Boccara, and D. Fournier, Appl. Opt. 20, 1333 (1981).

7. I. Murase, T. Ohnishi, T. Noguchi, and M. Hirooka, Polym. Comm. 25, 327 (1984).

8. K.F. Voss, C.M. Foster, L. Smilowitz, D. Mihailovic, S. Askari, G. Srdanov, Z. Ni, A.J. Heeger, and F. Wudl, Phys. Rev. B43, 5109 (1991).

9. C.H. Seager, M. Sinclair, D. McBranch, A.J. Heeger, and G.L. Baker, this volume.

10. T. Kaino, Jpn. J. Appl. Phys. 24, 1661 (1985).

11. S. Yamata, S. Tokito, T. Tsutsui, and S. Saito, J. Chem. Sci., Chem. Comm., 1448 , . nas 
device can result in a thermally induced refractive index change that could dominate the optically induced refractive index change. Optical absorption can also lead to damage or degradation of the nonlinear material. Finally, scattering of the guided optical wave could cause cross-talk between nearby guides.

Detailed investigations of the optical losses of the earliest conjugated polymers such as polyacetylene and polythiophene were largely impractical because films of these materials tended to be of very poor optical quality, making assessment of the relative influence of factors such as scattering and impurity absorption on experimental findings difficult. More recently, however, there has been an increased emphasis on processability of conjugated polymers, and as a result, there have been significant improvements in film quality. In particular, the development of materials which are soluble in organic solvents, coupled with the application of solution processing techniques (such as solution filtering, spin-casting, etc.), has led to the production of thin films of vastly improved quality [5]. With these better films, detailed studies of optical losses become possible. In this paper, we describe the results of Photothermal Deflection Spectroscopy measurements of the subgap absorption of solution processed conjugated polymers.

\section{EXPERIMENTAL}

In Photothermal Deflection Spectroscopy (PDS), the absorption of a material is determined by measuring the temperature rise of the sample when it is irradiated by an optical beam of known intensity [6]. To record the temperature rise, the sample is immersed in a liquid and the beam from a Helium-Neon laser is propagated parallel to the front surface of the material in the region illuminated by the heating beam. Any change in the temperature at the surface of the sample will result in the formation of a thermal gradient in the liquid immediately adjacent to the surface of the sample. Because the refractive index of the liquid is temperature dependent, the induced refractive index gradient will cause an angular deflection of the laser beam. The amount of heating light absorbed by the sample may be deduced by measuring the magnitude of this deflection, and comparing it to the deflection induced by a totally absorbing sample.

In practice, the samples were suspended in a $1 \mathrm{~cm}$ cuvette filled with Fluorinert (FC75). The beam from a stabilized He-Ne laser was focussed with a cylindrical lens so that the beam waist occured at the center of the cell. After traversing the sample cell, the angular deflection of the beam was measured using a knife-edge detector. In addition, a portion of the He-Ne beam was split off prior to the sample cell and directed onto an identical detector for use in reducing noise due to amplitude and beam pointing fluctuations of the laser. The heating beam was obtained using a tungsten-halogen lamp, mechanical chopper, and grating monochromator; and lock-in detection was used to measure the deflection of the $\mathrm{He}-\mathrm{Ne}$ beam. For further noise reduction, the entire apparatus was arranged on a vibration isolated table, and the sample chamber was enclosed in an air-tight box. The spectral range of our spectrometer extends from $0.55 \mathrm{eV}$ to $3 \mathrm{eV}$ with an absorptance sensitivity of $<10^{-5}$.

The choice of Fluorinert FC-75 was dictated by the need to use a liquid which is a nonsolvent for the (soluble) conjugated polymers, but which possesses a high degree of transparency in the spectral region of interest. The samples were prepared by spin-casting thin films onto fused silica substrates. Whenever possible, sample handling procedures were 
performed in inert environments. Prior to mounting the samples in the cuvette, helium was bubbled through the fluorinert to reduce the concentration of dissolved oxygen; and after mounting, the sample cell was hermetically sealed. Subsequent to measuring the absorptance spectra, the thickness of the samples were measured using a Sloan Dektak, and the absorption spectra were calculated.

\section{RESULTS AND DISCUSSION}

In general, the PDS spectra for a given polymer varied somewhat from sample to sample. This was particularly true in the initial stages of the experiment before it was realized that exposure to above band gap light (particularly in the presence of oxygen) can lead to significant alteration of the subgap absorption spectra. For subsequent samples, care was taken to minimize exposure to air and light. With reasonable care, the variations from sample to sample were typically no more than $\pm 25 \%$.

Fig. 1 displays the PDS spectra of a thin film of poly[1,4-phenylene-vinylene] (PPV). The film was prepared by depositing the precursor polymer from aqueous solution, followed by heating to convert it to PPV [7]. For PPV, the slope of the absorption edge is largest at the highest energies and has an abrupt change to a smaller slope at about $2.3 \mathrm{eV}$. Below this energy the absorption gradually decreases to a value of about $6 \mathrm{~cm}^{-1}$, which is the minimum absorption observed for this material. In the low energy portion of the spectrum, there is a pronounced peak at $0.74 \mathrm{eV}$ which we believe is the first overtone of the $\mathrm{C}-\mathrm{H}$ stretching mode $\left(\nu_{2}^{\mathrm{C}-\mathrm{H}}\right)$. The smaller peak at $0.88 \mathrm{eV}$ is due to the stretching plus bending combination overtone $\left(\nu_{2}^{\mathrm{C}-\mathrm{H}}+\delta^{\mathrm{C}-\mathrm{H}}\right)$ with some contribution from the first overtone of the substrate $\mathrm{O}-\mathrm{H}$ stretching mode $\left(\nu_{2}^{\mathrm{O}-\mathrm{H}}\right)$, since both of these absorptions are expected to appear at about the same energy.

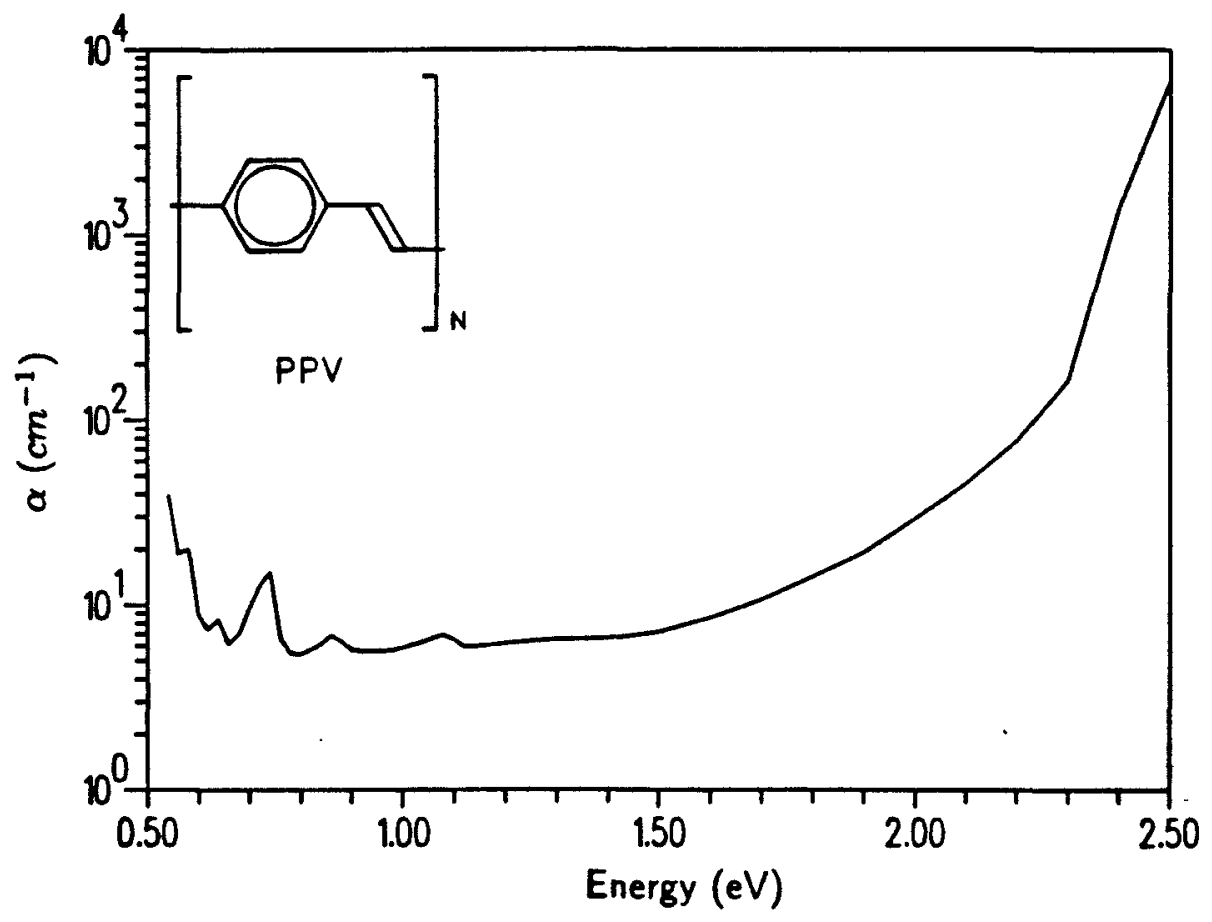

Fig. 1. Structure and PDS spectrum of PPV. 


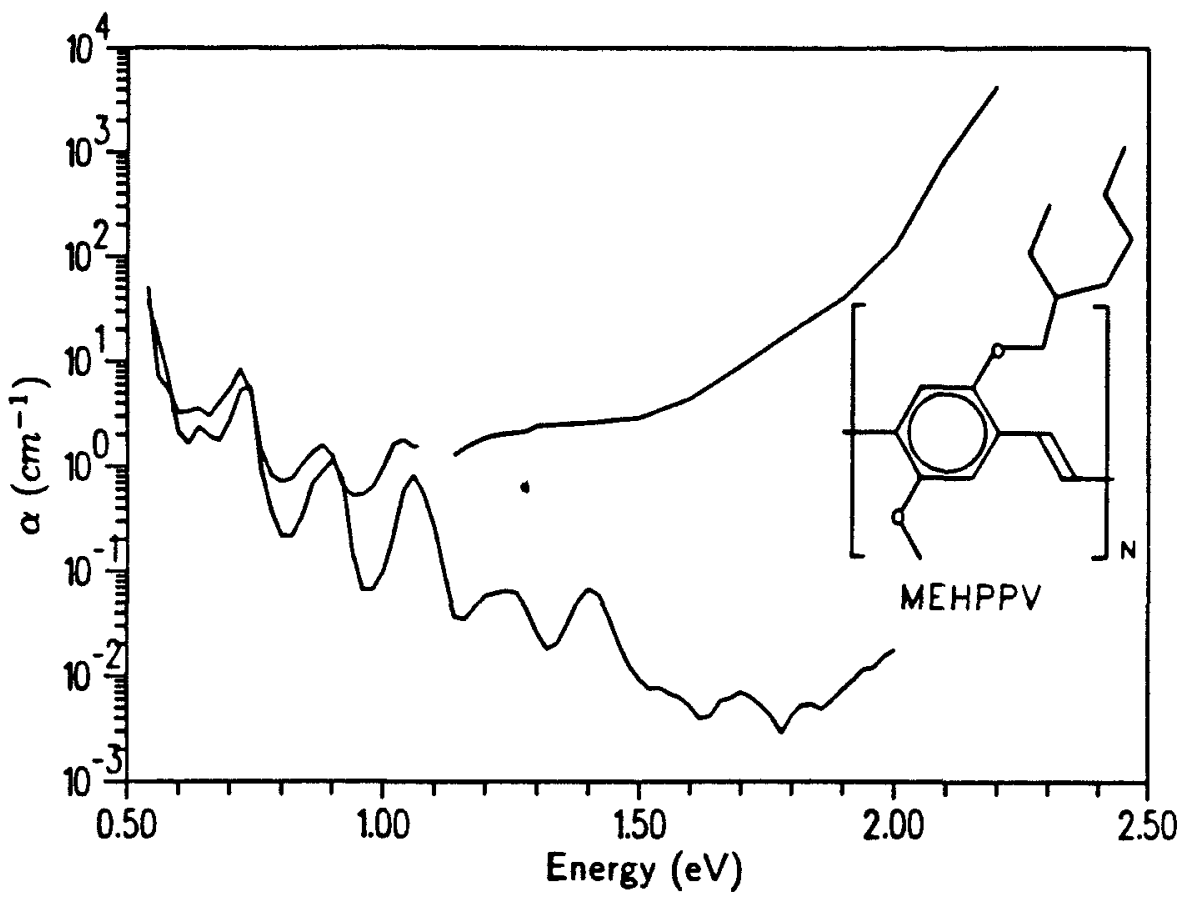

Fig. 2. Upper curve: PDS spectrum of MEHPPV. Lower curve: PDS spectrum of PMMA.

The upper curve in Fig. 2 is the PDS spectrum of a thin film of poly[2-methoxy, 5(2'-ethyl-hexoxy)-PPV] (MEHPPV) prepared by spin-casting from $m$-xylenes. The band edge of MEHPPV is red shifted by about $0.3 \mathrm{eV}$ relative to that of PPV due to the addition of the alkoxy side chains [8]. The minimum absorption coefficient observed for MEHPPV $\left(0.7 \mathrm{~cm}^{-1}\right)$ occurs at $0.95 \mathrm{eV}$, and, once again, overtone features appear in the low energy portion of the spectrum. An interesting feature of the PDS spectrum of MEHPPV is the broad featureless absorption which is visible as a shoulder at $1.3 \mathrm{eV}$. We believe that this is due to residual (long-lived) photoexcitations generated by light exposure during sample handling as well as the high energy portion of the PDS run. This observation is supported by the photoinduced absorption data presented in a companion paper [9], as well as the data of Voss et al. [8]. If this is indeed the case, then for maximum subgap transparency, samples of this material should be protected from photons with energy above the $\pi-\pi^{*}$ transition.

To further investigate the role of $\mathrm{C}-\mathrm{H}$ overtones, we measured the PDS spectrum of the nonconjugated polymer poly[methylmethacrylate] (lower curve in Fig. 2). In the nearinfrared, the absorption spectrum of this material is known to be dominated by $\mathrm{C}-\mathrm{H}$ overtones [10]. We have converted our raw absorptance data to an absorption spectrum by scaling our data so that the magnitude of the overtone absorption at $0.74 \mathrm{eV}$ agreed with the data of Kaino et al. [10]. The close similarity between the spectra of MEHPPV and PMMA in the spectral region below $1.1 \mathrm{eV}$, further supports the assignments of the $\mathrm{C}-\mathrm{H}$ overtone absorptions described above. From the PDS spectrum of PMMA, we can see that the magnitude of the $\mathrm{C}-\mathrm{H}$ absorption falls quickly with increasing photon energy, and in the spectral region from $1.1 \mathrm{eV}$ to $2 \mathrm{eV}, \mathrm{C}-\mathrm{H}$ absorption contributes less than $0.1 \mathrm{~cm}^{-1}$. Thus, we conclude that, although $\mathrm{C}-\mathrm{H}$ overtones are observable at low energies, the residual subgap absorption above $\sim 1 \mathrm{eV}$ in MEHPPV (and PPV) is dominated by other mechanisms. 


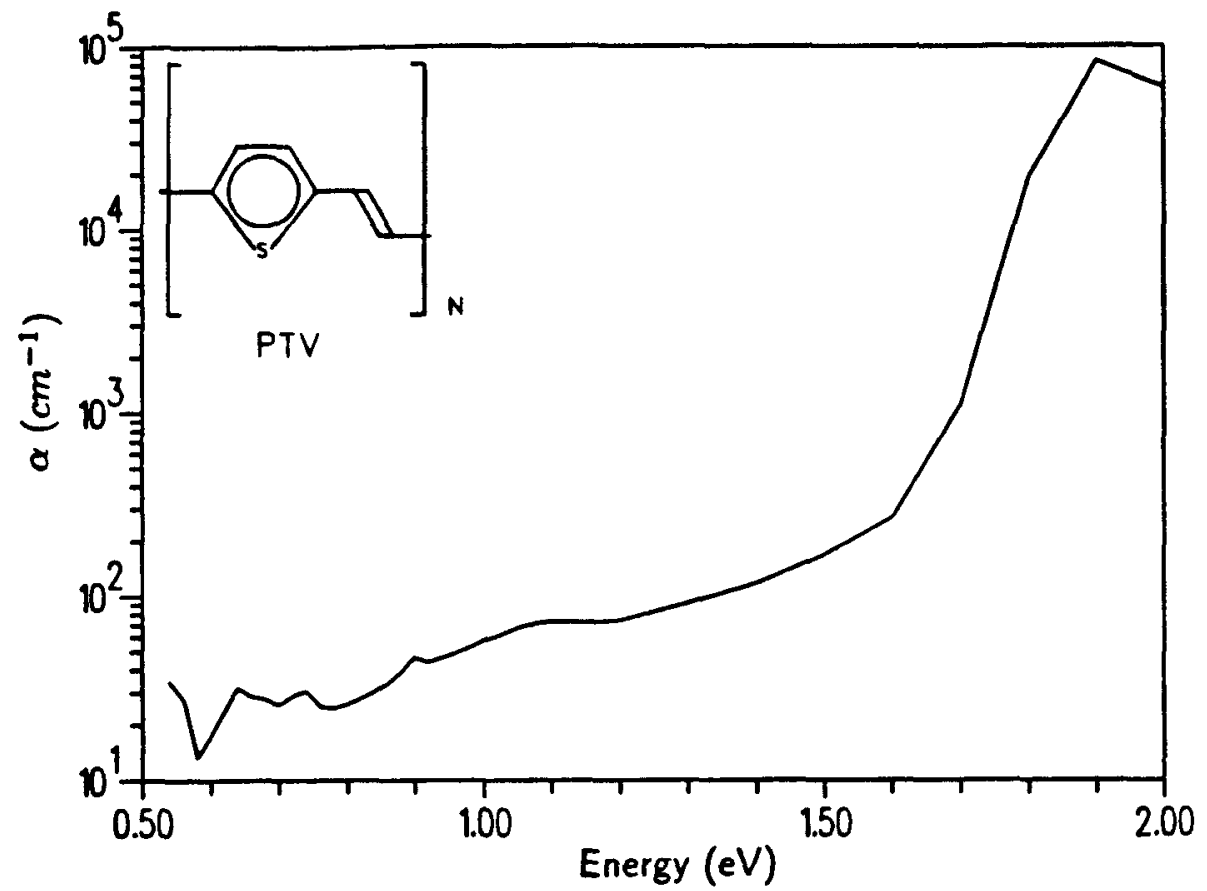

Fig. 3. Structure and PDS spectrum of PTV.

The PDS spectrum of poly[2,5-thienylene-vinylene] (PTV) is displayed in Fig. 3. As in the case of PPV, this film was prepared by solution deposition of a precursor polymer and subsequent conversion to the final form [11]. The absorption edge of PTV is significantly red shifted relative to that of $P P V$, and the minimum absorption observed is $20-30 \mathrm{~cm}^{-1}$. As in the case of MEHPPV, a broad subgap absorption peak is observed (this time occuring at about $1.1 \mathrm{eV}$ ) which may be due to residual photoexcitations generated in the sample handling and measurement process.

Finally, the subgap optical spectrum of a film of poly[5,7-dodecadiyne-1,12-diolbis ( $n$ butoxycarbonylmethylurethane)] (PDA-4BCMU), is presented in Fig. 4. This film was

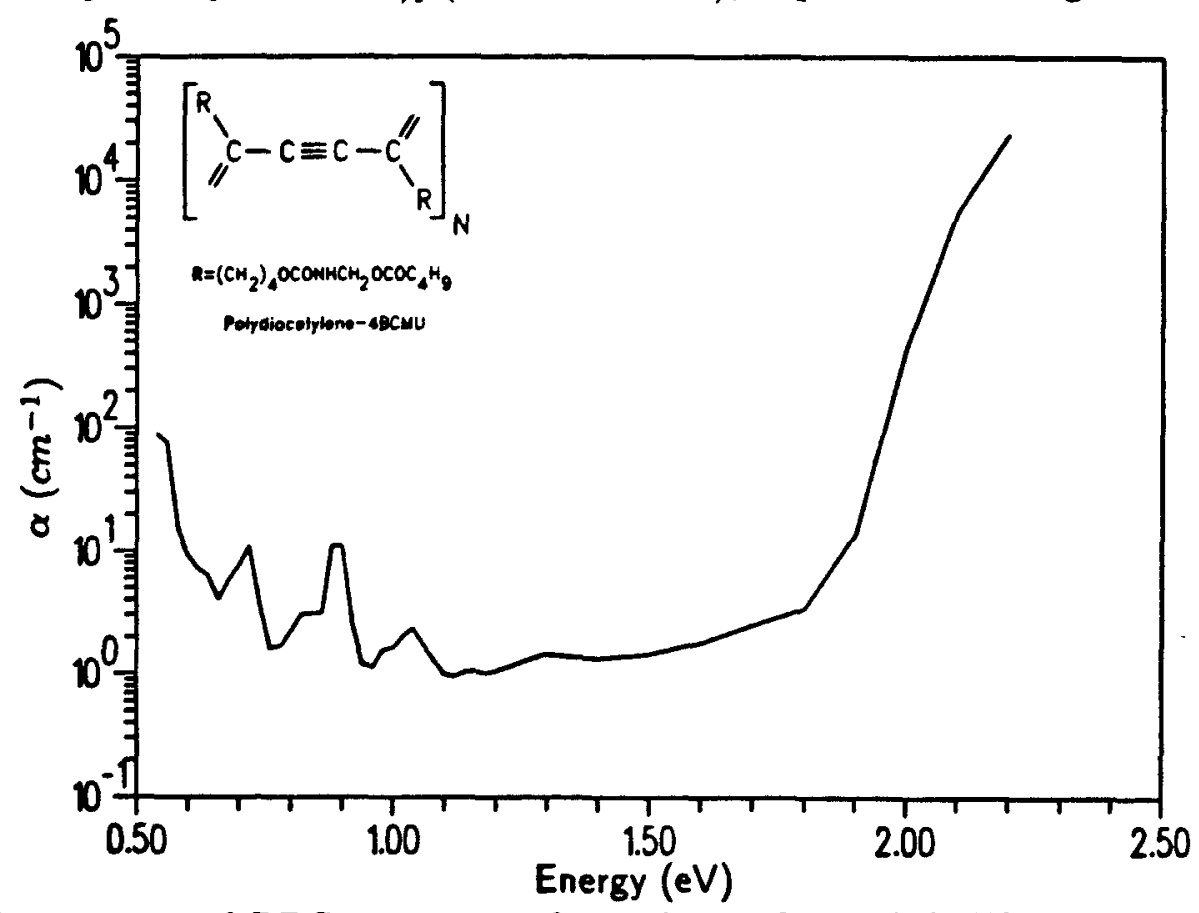

Fig. 4. Structure and PDS spectrum of polydiacetylene-4BCMU. 
prepared by spin-casting from cyclopentanone [5]. Of all the conjugated polymers we have measured, PDA-4BCMU possesses the sharpest absorption edge, with the absorption coefficient falling quickly to a value of about $1 \mathrm{~cm}^{-1}$. At lower energies, overtone features are again observed.

From these results, we conclude that the most serious source of subgap absorption in conjugated polymers is the low energy tail of the principle absorption edge, which decreases very slowly with decreasing photon energy. Although the origin of this tail is not presently known, the following mechanisms may contribute: inhomogeneous broadening due to a distribution of chain lengths and environments; large differences in ground and excited state geometries (i.e. vibronic effects); impurity absorption; interchain interactions; and the absorption tails of broad higher-lying absorptions. Current investigations are directed towards identifying the contribution of each of these mechanisms to the subgap absorption.

\section{CONCLUSION}

We have measured the subgap optical absorption of conjugated polymers using Photothermal Deflection Spectroscopy. At the lowest photon energies, absortions due to $\mathrm{C}-\mathrm{H}$ overtones are observed. By comparing with the $\mathrm{C}-\mathrm{H}$ overtone spectrum of PMMA, we conclude that for most integrated optical applications overtone absorption will not present a significant problem. In contrast, we find that the presence of a low energy tail of the principle absorption edge severely limits the transparency of the conjugated polymers we have measured. The minimum absorpion coefficients observed ranged from $1 \mathrm{~cm}^{-1}(\sim 4 \mathrm{~dB} / \mathrm{cm})$ to $10 \mathrm{~cm}^{-1}(40 \mathrm{~dB} / \mathrm{cm})$. The origin of this tail must be identified in order to continue to develop conjugated polymers for guided wave applications.

Acknowledgements: We thank W.R. Bayless for assistance in making the PDS measurements.

\section{REFERENCES}

1. Nonlinear Optical Properties of Polymers, edited by A.J. Heeger, J. Orenstein, and D. Ulrich (Mater. Res. Soc. Proc. 109, Pittsburgh, PA, 1988).

2. M. Thakur, R.C. Frye, and B.I. Greene, Appl. Phys. Lett. 56, 1187 (1990).

3. W. Krug, E. Miao, M. Derstine, and J. Valera, JOSA B6, 726 (1989).

4. P.D. Townsend, W.-S. Fann, S. Etemad, G.L. Baker, Z. G. Zoos, and P.C.M. McWilliams, (Chem. Phys. Lett., submitted).

5. P.D. Townsend, G.L. Baker, N.E. Sclotter, C.F. Klausner, and S. Etemad, Appl. Phys. Lett. 53, 1782, (1988).

6. W.B. Jackson, N. Amer, A.C. Boccara, and D. Fournier, Appl. Opt. 20, 1333 (1981).

7. I. Murase, T. Ohnishi, T. Noguchi, and M. Hirooka, Polym. Comm. 25, 327 (1984).

8. K.F. Voss, C.M. Foster, L. Smilowitz, D. Mihailovic, S. Askari, G. Srdanov, Z. Ni, A.J. Heeger, and F. Wudl, Phys. Rev. B43, 5109 (1991).

9. C.H. Seager, M. Sinclair, D. McBranch, A.J. Heeger, and G.L. Baker, this volume.

10. T. Kaino, Jpn. J. Appl. Phys. 24, 1661 (1985).

11. S. Yamata, S. Tokito, T. Tsutsui, and S. Saito, J. Chem. Sci., Chem. Comm., 1448 $\ldots \ldots$ 